Acta Arith. 180(2017), no. 3, 229-249.

\title{
SUMS OF FOUR POLYGONAL NUMBERS WITH COEFFICIENTS
}

\author{
XIANG-ZI MENG AND ZHI-WEI SUN
}

\begin{abstract}
Let $m \geqslant 3$ be an integer. The polygonal numbers of order $m+2$ are given by $p_{m+2}(n)=m\left(\begin{array}{c}n \\ 2\end{array}\right)+n(n=0,1,2, \ldots)$. A famous claim of Fermat proved by Cauchy asserts that each nonnegative integer is the sum of $m+2$ polygonal numbers of order $m+2$. For $(a, b)=$ $(1,1),(2,2),(1,3),(2,4)$, we study whether any sufficiently large integer can be expressed as

$$
p_{m+2}\left(x_{1}\right)+p_{m+2}\left(x_{2}\right)+a p_{m+2}\left(x_{3}\right)+b p_{m+2}\left(x_{4}\right)
$$

with $x_{1}, x_{2}, x_{3}, x_{4}$ nonnegative integers. We show that the answer is positive if $(a, b) \in\{(1,3),(2,4)\}$, or $(a, b)=(1,1) \& 4 \mid m$, or $(a, b)=$ $(2,2) \& m \not \equiv 2(\bmod 4)$. In particular, we confirm a conjecture of Z.-W. Sun which states that any natural number can be written as $p_{6}\left(x_{1}\right)+$ $p_{6}\left(x_{2}\right)+2 p_{6}\left(x_{3}\right)+4 p_{6}\left(x_{4}\right)$ with $x_{1}, x_{2}, x_{3}, x_{4}$ nonnegative integers.
\end{abstract}

\section{InTRODUCTION}

Let $m \in \mathbb{Z}^{+}=\{1,2,3, \ldots\}$. The polygonal numbers of order $m+2$ (or $(m+2)$-gonal numbers), which are constructed geometrically from the regular polygons with $m+2$ sides, are given by

$$
p_{m+2}(n):=m\left(\begin{array}{l}
n \\
2
\end{array}\right)+n=\frac{m n^{2}-(m-2) n}{2} \text { for } n \in \mathbb{N}=\{0,1,2, \ldots\} .
$$

Clearly,

$$
p_{m+2}(0)=0, p_{m+2}(1)=1, p_{m+2}(2)=m+2, p_{m+2}(3)=3 m+3,
$$

and $p_{m+2}(x)$ with $x \in \mathbb{Z}$ are called generalized $(m+2)$-gonal numbers. It is easy to see that generalized hexagonal numbers coincide with triangular numbers (i.e., those $p_{3}(n)=n(n+1) / 2$ with $n \in \mathbb{N}$ ). Note that

$$
p_{4}(n)=n^{2}, p_{5}(n)=\frac{n(3 n-1)}{2}, p_{6}(n)=n(2 n-1)=p_{3}(2 n-1) .
$$

Fermat's claim that each $n \in \mathbb{N}$ can be written as the sum of $m+2$ polygonal numbers of order $m+2$ was proved by Lagrange in the case $m=2$, by Gauss in the case $m=1$, and by Cauchy in the case $m \geqslant 3$ (cf. [9, pp. 3-35] and [7,

2010 Mathematics Subject Classification. Primary 11E20, 11E25; Secondary 11B13, 11B75, 11D85, 11P99.

Key words and phrases. Polygonal numbers, additive bases, ternary quadratic forms. 
pp. 54-57]). In 1830 Legendre refined Cauchy's polygonal number theorem by showing that any integer $N \geqslant 28 m^{3}$ with $m \geqslant 3$ can be written as

$$
p_{m+2}\left(x_{1}\right)+p_{m+2}\left(x_{2}\right)+p_{m+2}\left(x_{3}\right)+p_{m+2}\left(x_{4}\right)+\delta_{m}(N)
$$

where $x_{1}, x_{2}, x_{3}, x_{4} \in \mathbb{N}, \delta_{m}(N)=0$ if $2 \nmid m$, and $\delta_{m}(N) \in\{0,1\}$ if 2 $m$. Nathanson ([8] and [9, p.33]) simplified the proofs of Cauchy's and Legendre's theorems.

In 1917 Ramanujan [10] listed 55 possible quadruples $(a, b, c, d)$ of positive integers with $a \leqslant b \leqslant c \leqslant d$ such that any $n \in \mathbb{N}$ can be written as $a x^{2}+b y^{2}+c z^{2}+d w^{2}$ with $x, y, z, w \in \mathbb{Z}$, and 54 of them were later confirmed by Dickson [2] while the remaining one on the list was actually wrong.

Recently, Sun [12] showed that any positive integer can be written as the sum of four generalized octagonal numbers one of which is odd. He also proved that for many triples $(b, c, d)$ of positive integers (including $(1,1,3)$, $(1,2,2)$ and $(1,2,4))$ we have

$$
\left\{p_{8}\left(x_{1}\right)+b p_{8}\left(x_{2}\right)+c p_{8}\left(x_{3}\right)+d p_{8}\left(x_{4}\right): x_{1}, x_{2}, x_{3}, x_{4} \in \mathbb{Z}\right\}=\mathbb{N} .
$$

In [12, Conjecture 5.3], Sun conjectured that any $n \in \mathbb{N}$ can be written as $p_{6}\left(x_{1}\right)+p_{6}\left(x_{2}\right)+2 p_{6}\left(x_{3}\right)+4 p_{6}\left(x_{4}\right)$ with $x_{1}, x_{2}, x_{3}, x_{4} \in \mathbb{N}$.

Motivated by the above work, for $(a, b)=(1,1),(2,2),(1,3),(2,4)$ and $m \in\{3,4,5, \ldots\}$, we study whether any sufficiently large integer can be written as

$$
p_{m+2}\left(x_{1}\right)+p_{m+2}\left(x_{2}\right)+a p_{m+2}\left(x_{3}\right)+b p_{m+2}\left(x_{4}\right) \text { with } x_{1}, x_{2}, x_{3}, x_{4} \in \mathbb{N} \text {. }
$$

Now we state our main results.

Theorem 1.1. Let $m \in \mathbb{Z}^{+}$with $4 \mid m$.

(i) Any integer $N \geqslant 28 \mathrm{~m}^{3}$ can be expressed as

$$
p_{m+2}\left(x_{1}\right)+p_{m+2}\left(x_{2}\right)+p_{m+2}\left(x_{3}\right)+p_{m+2}\left(x_{4}\right) \quad\left(x_{1}, x_{2}, x_{3}, x_{4} \in \mathbb{N}\right) .
$$

(ii) There are infinitely many positive integers not of the form $p_{m+4}\left(x_{1}\right)+$ $p_{m+4}\left(x_{2}\right)+p_{m+4}\left(x_{3}\right)+p_{m+4}\left(x_{4}\right)$ with $x_{1}, x_{2}, x_{3}, x_{4} \in \mathbb{N}$.

Remark 1.1. This can be viewed as a supplement to Legendre's theorem. By Theorem 1.1(ii), there are infinitely many positive integers none of which is the sum of four octagonal numbers; in contrast, Sun [12] showed that any $n \in \mathbb{N}$ is the sum of four generalized octagonal numbers. 
Corollary 1.1. We have

$$
\begin{aligned}
& \left\{p_{6}\left(x_{1}\right)+p_{6}\left(x_{2}\right)+p_{6}\left(x_{3}\right)+p_{6}\left(x_{4}\right): x_{1}, x_{2}, x_{3}, x_{4} \in \mathbb{N}\right\} \\
= & \mathbb{N} \backslash\{5,10,11,20,25,26,38,39,54,65,70,114,130\}
\end{aligned}
$$

and hence any $n \in \mathbb{N}$ can be written as the sum of a triangular number and three hexagonal numbers. Also, any integer $n>2146$ can be written as the sum of four decagonal numbers and thus

$\left\{p_{10}\left(x_{1}\right)+p_{10}\left(x_{2}\right)+p_{10}\left(x_{3}\right)+p_{10}\left(x_{4}\right): x_{1}, x_{2}, x_{3}, x_{4} \in \mathbb{Z}\right\}=\mathbb{N} \backslash\{5,6,26\}$.

Proof. Via a computer, we can easily verify that

$$
5,10,11,20,25,26,38,39,54,65,70,114,130
$$

are the only natural numbers smaller than $28 \times 4^{3}$ which cannot be written as the sum of four hexagonal numbers, but all these numbers can be expressed as the sum of a triangular number and three hexagonal numbers. Also, every $n=2147, \ldots, 28 \times 8^{3}-1$ is the sum of four decagonal numbers, and 5, 6 and 26 are the only natural numbers smaller than 2147 which cannot be written as the sum of four generalized decagonal numbers. Now it suffices to apply Theorem 1.1 with $m=4,8$.

Remark 1.2. Sun [11, Conjecture 1.10] conjectured that any $n \in \mathbb{N}$ can be written as the sum of two triangular numbers and a hexagonal number. Krachun [6] proved that

$$
\begin{aligned}
& \left\{p_{6}(-w)+p_{6}(-x)+p_{6}(y)+p_{6}(z): w, x, y, z \in \mathbb{N}\right\} \\
= & \left\{p_{6}(-w)+2 p_{6}(-x)+p_{6}(y)+2 p_{6}(z): w, x, y, z \in \mathbb{N}\right\}=\mathbb{N},
\end{aligned}
$$

which was first conjectured by the second author [12].

Theorem 1.2. Let $m \geqslant 3$ be an integer.

(i) Suppose that $2 \nmid m$ or $4 \mid m$. Then any integer $N \geqslant 1628 m^{3}$ can be written as

$$
p_{m+2}\left(x_{1}\right)+p_{m+2}\left(x_{2}\right)+2 p_{m+2}\left(x_{3}\right)+2 p_{m+2}\left(x_{4}\right) \quad\left(x_{1}, x_{2}, x_{3}, x_{4} \in \mathbb{N}\right) .
$$

(ii) If $m \equiv 2(\bmod 4)$, then there are infinitely many positive integers not represented by $p_{m+2}\left(x_{1}\right)+p_{m+2}\left(x_{2}\right)+2 p_{m+2}\left(x_{3}\right)+2 p_{m+2}\left(x_{4}\right)$ with $x_{1}, x_{2}, x_{3}, x_{4} \in \mathbb{N}$.

Remark 1.3. Actually our proof of Theorem 1.2(i) given in Section 3 allows us to replace $1628 m^{3}$ by $418 m^{3}$ in the case $m \equiv 1(\bmod 2)$. By Theorem 
1.2(ii), there are infinitely many positive integers not represented by $p_{8}\left(x_{1}\right)+$ $p_{8}\left(x_{2}\right)+2 p_{8}\left(x_{3}\right)+2 p_{8}\left(x_{4}\right)$ with $x_{1}, x_{2}, x_{3}, x_{4} \in \mathbb{N}$; in contrast, Sun [12] proved that any $n \in \mathbb{N}$ can be written as $p_{8}\left(x_{1}\right)+p_{8}\left(x_{2}\right)+2 p_{8}\left(x_{3}\right)+2 p_{8}\left(x_{4}\right)$ with $x_{1}, x_{2}, x_{3}, x_{4} \in \mathbb{Z}$.

Corollary 1.2. We have

$$
\begin{gathered}
\left\{p_{5}\left(x_{1}\right)+p_{5}\left(x_{2}\right)+2 p_{5}\left(x_{3}\right)+2 p_{5}\left(x_{4}\right): x_{1}, x_{2}, x_{3}, x_{4} \in \mathbb{N}\right\}=\mathbb{N}, \\
\left\{p_{6}\left(x_{1}\right)+p_{6}\left(x_{2}\right)+2 p_{6}\left(x_{3}\right)+2 p_{6}\left(x_{4}\right): x_{1}, x_{2}, x_{3}, x_{4} \in \mathbb{N}\right\} \\
=\mathbb{N} \backslash\{22,82,100\}
\end{gathered}
$$

and

$$
\begin{aligned}
& \left\{p_{7}\left(x_{1}\right)+p_{7}\left(x_{2}\right)+2 p_{7}\left(x_{3}\right)+2 p_{7}\left(x_{4}\right): x_{1}, x_{2}, x_{3}, x_{4} \in \mathbb{N}\right\} \\
& \quad=\mathbb{N} \backslash\{13,26,31,65,67,173,175,215,247\} .
\end{aligned}
$$

Also, for each $k=9,10,11$, any integer $n>C_{k}$ can be written as $p_{k}\left(x_{1}\right)+$ $p_{k}\left(x_{2}\right)+2 p_{k}\left(x_{3}\right)+2 p_{k}\left(x_{4}\right)$ with $x_{1}, x_{2}, x_{3}, x_{4} \in \mathbb{N}$, where $C_{9}=925, C_{10}=840$ and $C_{11}=1799$. Therefore,

$$
\begin{array}{r}
\left\{p_{3}(w)+p_{6}(x)+2 p_{6}(y)+2 p_{6}(z): w, x, y, z \in \mathbb{N}\right\}=\mathbb{N}, \\
\left\{2 p_{3}(w)+p_{6}(x)+p_{6}(y)+2 p_{6}(z): w, x, y, z \in \mathbb{N}\right\}=\mathbb{N}, \\
\left\{p_{7}(w)+p_{7}(x)+2 p_{7}(y)+2 p_{7}(z): w \in \mathbb{Z} \& x, y, z \in \mathbb{N}\right\}=\mathbb{N}, \\
\left\{p_{9}(w)+2 p_{9}(x)+p_{9}(y)+2 p_{9}(z): w, x \in \mathbb{Z} \& y, z \in \mathbb{N}\right\}=\mathbb{N}, \\
\left\{p_{10}(w)+2 p_{10}(x)+p_{10}(y)+2 p_{10}(z): w, x \in \mathbb{Z} \& y, z \in \mathbb{N}\right\}=\mathbb{N},
\end{array}
$$

and

$$
\left\{p_{11}(w)+2 p_{11}(x)+p_{11}(y)+2 p_{11}(z): w, x \in \mathbb{Z} \& y, z \in \mathbb{N}\right\}=\mathbb{N} \backslash\{7\} .
$$

Proof. Note that $\left\{p_{6}(w): w \in \mathbb{Z}\right\}=\left\{p_{3}(w): w \in \mathbb{N}\right\}$. It suffices to apply Theorem $1.2(\mathrm{i})$ with $m \in\{3,4,5,7,8,9\}$ and check those $n \in \mathbb{N}$ with $n<1628 m^{3}$ via a computer.

Remark 1.4. (1.6) appeared as part of [12, Conjecture 5.2(ii)], and it indicates that the set $\left\{p_{5}(x)+2 p_{5}(y): x, y \in \mathbb{N}\right\}$ is an additive base of order 2 . For positive integers $a, b, c$ with $\left\{a p_{5}(x)+b p_{5}(y)+c p_{5}(z): x, y, z \in \mathbb{Z}\right\}=\mathbb{N}$, see [11] and [4].

Theorem 1.3. Let $m \geqslant 3$ be an integer. Then each integer $N \geqslant 924 m^{3}$ can be expressed as

$$
p_{m+2}\left(x_{1}\right)+p_{m+2}\left(x_{2}\right)+p_{m+2}\left(x_{3}\right)+3 p_{m+2}\left(x_{4}\right) \quad\left(x_{1}, x_{2}, x_{3}, x_{4} \in \mathbb{N}\right) .
$$


Corollary 1.3. We have

$$
\begin{gathered}
\left\{p_{5}\left(x_{1}\right)+p_{5}\left(x_{2}\right)+p_{5}\left(x_{3}\right)+3 p_{5}\left(x_{4}\right): x_{1}, x_{2}, x_{3}, x_{4} \in \mathbb{N}\right\}=\mathbb{N} \backslash\{19\} \\
\left\{p_{6}\left(x_{1}\right)+p_{6}\left(x_{2}\right)+p_{6}\left(x_{3}\right)+3 p_{6}\left(x_{4}\right): x_{1}, x_{2}, x_{3}, x_{4} \in \mathbb{N}\right\} \\
=\mathbb{N} \backslash\{14,23,41,42,83\}
\end{gathered}
$$

and

$$
\begin{aligned}
& \left\{p_{7}\left(x_{1}\right)+p_{7}\left(x_{2}\right)+p_{7}\left(x_{3}\right)+3 p_{7}\left(x_{4}\right): x_{1}, x_{2}, x_{3}, x_{4} \in \mathbb{N}\right\} \\
= & \mathbb{N} \backslash\{13,16,27,31,33,49,50,67,87,178,181,259\} .
\end{aligned}
$$

Also, for each $k=8,9,10$, any integer $n>M_{k}$ can be written as $p_{k}\left(x_{1}\right)+$ $p_{k}\left(x_{2}\right)+p_{k}\left(x_{3}\right)+3 p_{k}\left(x_{4}\right)$ with $x_{1}, x_{2}, x_{3}, x_{4} \in \mathbb{N}$, where $M_{8}=435, M_{9}=695$ and $M_{10}=916$. Therefore

$$
\begin{aligned}
& \left\{p_{7}(w)+p_{7}(x)+p_{7}(y)+3 p_{7}(z): w \in \mathbb{Z} \& x, y, z \in \mathbb{N}\right\}=\mathbb{N}, \\
& \left\{p_{9}\left(x_{1}\right)+p_{9}\left(x_{2}\right)+p_{9}\left(x_{3}\right)+3 p_{9}\left(x_{4}\right): x_{1}, x_{2}, x_{3}, x_{4} \in \mathbb{Z}\right\}=\mathbb{N} \backslash\{17\},
\end{aligned}
$$

$$
\left\{p_{10}\left(x_{1}\right)+p_{10}\left(x_{2}\right)+p_{10}\left(x_{3}\right)+3 p_{10}\left(x_{4}\right): x_{1}, x_{2}, x_{3}, x_{4} \in \mathbb{Z}\right\}=\mathbb{N} \backslash\{16,19\} .
$$

Proof. It suffices to apply Theorem 1.3 with $m \in\{3,4,5,6,7,8\}$ and check those $n \in \mathbb{N}$ with $n<924 m^{3}$ via a computer.

Remark 1.5. Guy [5] thought that 10, 16 and 76 might be the only natural numbers which cannot be written as the sum of three generalized heptagonal numbers. The second author [12, Remark 5.2 and Conjecture 1.2] conjectured that $\left\{p_{7}(x)+p_{7}(y)+p_{7}(z): x, y, z \in \mathbb{Z}\right\}=\mathbb{N} \backslash\{10,16,76,307\}$ and

$$
\left\{p_{8}(x)+p_{8}(y)+3 p_{8}(z): x, y, z \in \mathbb{Z}\right\}=\mathbb{N} \backslash\{7,14,18,91\} .
$$

Theorem 1.4. Let $m \geqslant 3$ be an integer. Then any integer $N \geqslant 1056 m^{3}$ can be written as

$$
p_{m+2}\left(x_{1}\right)+p_{m+2}\left(x_{2}\right)+2 p_{m+2}\left(x_{3}\right)+4 p_{m+2}\left(x_{4}\right) \quad\left(x_{1}, x_{2}, x_{3}, x_{4} \in \mathbb{N}\right) .
$$

Corollary 1.4. We have

$$
\begin{aligned}
& \left\{p_{5}\left(x_{1}\right)+p_{5}\left(x_{2}\right)+2 p_{5}\left(x_{3}\right)+4 p_{5}\left(x_{4}\right): x_{1}, x_{2}, x_{3}, x_{4} \in \mathbb{N}\right\}=\mathbb{N} \\
& \left\{p_{6}\left(x_{1}\right)+p_{6}\left(x_{2}\right)+2 p_{6}\left(x_{3}\right)+4 p_{6}\left(x_{4}\right): x_{1}, x_{2}, x_{3}, x_{4} \in \mathbb{N}\right\}=\mathbb{N} \\
& \left\{p_{7}\left(x_{1}\right)+p_{7}\left(x_{2}\right)+2 p_{7}\left(x_{3}\right)+4 p_{7}\left(x_{4}\right): x_{1}, x_{2}, x_{3}, x_{4} \in \mathbb{N}\right\}=\mathbb{N} \backslash\{17,51\} \\
& \left\{p_{8}\left(x_{1}\right)+p_{8}\left(x_{2}\right)+2 p_{8}\left(x_{3}\right)+4 p_{8}\left(x_{4}\right): x_{1}, x_{2}, x_{3}, x_{4} \in \mathbb{N}\right\} \\
& \quad=\mathbb{N} \backslash\{19,30,39,59,78,91\}
\end{aligned}
$$


and

$$
\begin{aligned}
& \left\{p_{9}\left(x_{1}\right)+p_{9}\left(x_{2}\right)+2 p_{9}\left(x_{3}\right)+4 p_{9}\left(x_{4}\right): x_{1}, x_{2}, x_{3}, x_{4} \in \mathbb{N}\right\} \\
= & \mathbb{N} \backslash\{17,21,34,41,44,67,89,104,119,170,237,245,290\} .
\end{aligned}
$$

Also, for each $k=10,11,12$ any integer $n>N_{k}$ can be written as $p_{k}\left(x_{1}\right)+$ $p_{k}\left(x_{2}\right)+2 p_{k}\left(x_{3}\right)+4 p_{k}\left(x_{4}\right)$ with $x_{1}, x_{2}, x_{3}, x_{4} \in \mathbb{N}$, where $N_{10}=333, N_{11}=$ 734 and $N_{12}=1334$. Therefore,

$$
\left\{p_{k}(w)+p_{k}(x)+2 p_{k}(y)+4 p_{k}(z): w \in \mathbb{Z} \& x, y, z \in \mathbb{N}\right\}=\mathbb{N}
$$

for $k=7,9$, and

$$
\left\{p_{k}(w)+p_{k}(x)+2 p_{k}(y)+4 p_{k}(z): w, x, y, z \in \mathbb{Z}\right\}=\mathbb{N}
$$

for $k=8,10,11,12$.

Proof. It suffices to apply Theorem 1.4 with $m \in\{3, \ldots, 10\}$ and check those $n \in \mathbb{N}$ with $n<1056 m^{3}$ via a computer.

Remark 1.6. (1.23) and (1.24) were first conjectured by the second author [12, Conjecture 5.2(ii) and Conjecture 5.3]. Sun [12, Remark 5.2] also conjectured that

$$
\left\{p_{7}(x)+2 p_{7}(y)+4 p_{7}(z): x, y, z \in \mathbb{Z}\right\}=\mathbb{N} \backslash\{131,146\} .
$$

We will show Theorems 1.1-1.4 in Sections 2-5 respectively.

Throughout this paper, for a prime $p$ and $a, n \in \mathbb{N}$, by $p^{a} \| n$ we mean $p^{a} \mid n$ and $p^{a+1} \nmid n$. For example, $4 \| n$ if and only if $n \equiv 4(\bmod 8)$.

\section{Proof of Theorem 1.1}

We first give a lemma which is a slight variant of [9, Lemma 1.10].

Lemma 2.1. Let $l, m, N \in \mathbb{Z}^{+}$with $N \geqslant 7 l^{2} m^{3}$. Then the length of the interval

$$
I_{1}=\left[\frac{1}{2}+\sqrt{\frac{6 N}{m}-3}, \frac{2}{3}+\sqrt{\frac{8 N}{m}}\right]
$$

is greater than $\mathrm{lm}$.

Proof. Let $L_{1}$ denote the length of the interval $I_{1}$. Then $L_{1}=\sqrt{8 x}-$ $\sqrt{6 x-3}+1 / 6$, where $x=N / m \geqslant 7 l^{2} m^{2}$. Let $l_{0}=l m-1 / 6$. Then

$$
\begin{aligned}
L_{1}>l m & \Longleftrightarrow \sqrt{8 x}>\sqrt{6 x-3}+l_{0} \\
& \Longleftrightarrow 2 x+3-l_{0}^{2}>2 l_{0} \sqrt{6 x-3} \\
& \Longleftrightarrow 4 x\left(x+3-7 l_{0}^{2}\right)+\left(l_{0}^{2}-3\right)^{2}+12 l_{0}^{2}>0
\end{aligned}
$$


As $x \geqslant 7 l_{0}^{2}$, by the above we have $L_{1}>l m$. This completes the proof.

The following lemma is a slight modification of [9, Lemma 1.11].

Lemma 2.2. Let $a, b, m, N \in \mathbb{Z}^{+}$with $m \geqslant 3$ and

$$
N=\frac{m}{2}(a-b)+b \geqslant \frac{2}{3} m \text {. }
$$

Suppose that $b$ belongs to the interval $I_{1}$ given by (2.1). Then

$$
b^{2}<4 a \text { and } 3 a<b^{2}+2 b+4 .
$$

Proof. Observe that

$$
a=\left(1-\frac{2}{m}\right) b+\frac{2 N}{m}
$$

and

$$
\begin{aligned}
b^{2}-4 a & =b^{2}-4\left(1-\frac{2}{m}\right) b-\frac{8 N}{m} \\
& =\left(b-2\left(1-\frac{2}{m}\right)\right)^{2}-4\left(\left(1-\frac{2}{m}\right)^{2}+\frac{2 N}{m}\right) .
\end{aligned}
$$

As $m \geqslant 3$ and $b \in I_{1}$, we have

$$
b \leqslant \frac{2}{3}+\sqrt{\frac{8 N}{m}}<2\left(1-\frac{2}{m}\right)+2 \sqrt{\left(1-\frac{2}{m}\right)^{2}+\frac{2 N}{m}}
$$

and hence $b^{2}-4 a<0$. On the other hand, since $(1 / 2-3 / m)^{2}<1$ and $b \in I_{1}$ we have

$$
b \geqslant \frac{1}{2}+\sqrt{\frac{6 N}{m}-3}>\frac{1}{2}-\frac{3}{m}+\sqrt{\left(\frac{1}{2}-\frac{3}{m}\right)^{2}-4+\frac{6 N}{m}}
$$

and hence

$$
\begin{aligned}
b^{2}+2 b+4-3 a & =b^{2}-\left(1-\frac{6}{m}\right) b+\left(4-\frac{6 N}{m}\right) \\
& =\left(b-\left(\frac{1}{2}-\frac{3}{m}\right)\right)^{2}-\left(\left(\frac{1}{2}-\frac{3}{m}\right)^{2}-4+\frac{6 N}{m}\right)
\end{aligned}
$$$$
>0 \text {. }
$$

This proves (2.2).

Lemma 2.3. Let $a, b, c$ be positive integers and let $x, y, z$ be real numbers. Then we have the inequality

$$
(a x+b y+c z)^{2} \leqslant(a+b+c)\left(a x^{2}+b y^{2}+c z^{2}\right) .
$$


Proof. By the Cauchy-Schwarz inequality (cf. [9, p. 178]),

$$
\begin{aligned}
& (\sqrt{a}(\sqrt{a} x)+\sqrt{b}(\sqrt{b} y)+\sqrt{c}(\sqrt{c} z))^{2} \\
\leqslant & \left((\sqrt{a})^{2}+(\sqrt{b})^{2}+(\sqrt{c})^{2}\right)\left((\sqrt{a} x)^{2}+(\sqrt{b} y)^{2}+(\sqrt{c} z)^{2}\right) .
\end{aligned}
$$

This yields the desired (2.3).

The following lemma with $2 \nmid a b$ is usually called Cauchy's Lemma (cf. [9, pp. 31-34]).

Lemma 2.4. Let $a$ and $b$ be positive integers satisfying (2.2). Suppose that $2 \nmid a b$, or $2 \| a$ and $2 \mid b$. Then there exist $s, t, u, v \in \mathbb{N}$ such that

$$
a=s^{2}+t^{2}+u^{2}+v^{2} \text { and } b=s+t+u+v .
$$

Proof. By the Gauss-Legendre theorem (cf. [9, Section 1.5]), we have

$$
\left\{x^{2}+y^{2}+z^{2}: x, y, z \in \mathbb{Z}\right\}=\mathbb{N} \backslash\left\{4^{k}(8 l+7): k, l \in \mathbb{N}\right\}
$$

We claim that there are $x, y, z \in \mathbb{Z}$ with $4 a-b^{2}=x^{2}+y^{2}+z^{2}$ such that all the numbers

$$
\begin{aligned}
& s=\frac{b+x+y+z}{4}, \quad t=\frac{b+x-y-z}{4} \\
& u=\frac{b-x+y-z}{4}, \quad v=\frac{b-x-y+z}{4}
\end{aligned}
$$

are integers.

Case 1. $2 \nmid a b$.

In this case, $4 a-b^{2} \equiv 3(\bmod 8)$ and hence $4 a-b^{2}=x^{2}+y^{2}+z^{2}$ for some $x, y, z \in \mathbb{Z}$ with $2 \nmid x y z$. Without loss of generality, we may assume that $x \equiv y \equiv z \equiv b(\bmod 4)$. (If $x \equiv-b(\bmod 4)$ then $-x \equiv b(\bmod 4)$.) Thus the numbers in (2.6) are all integers.

Case 2. $2 \| a$ and $2 \mid b$.

Write $a=2 a_{0}$ and $b=2 b_{0}$ with $a_{0}, b_{0} \in \mathbb{Z}$ and $2 \nmid a_{0}$. Since $2 a_{0}-b_{0}^{2} \equiv$ $1,2(\bmod 4)$, we have $2 a_{0}-b_{0}^{2}=x_{0}^{2}+y_{0}^{2}+z_{0}^{2}$ for some $x_{0}, y_{0}, z_{0} \in \mathbb{Z}$. Without loss of generality, we may assume that $x_{0} \equiv b_{0}(\bmod 2)$ and $y_{0} \equiv z_{0}(\bmod 2)$. Set $x=2 x_{0}, y=2 y_{0}$ and $z=2 z_{0}$. Then $4 a-b^{2}=4\left(2 a_{0}-b_{0}^{2}\right)=x^{2}+y^{2}+z^{2}$, and the numbers in (2.6) are all integers. 
In either case, there are $x, y, z \in \mathbb{Z}$ for which $4 a-b^{2}=x^{2}+y^{2}+z^{2}$ and $s, t, u, v \in \mathbb{Z}$, where $s, t, u, v$ are as in (2.6). Obviously, $s+t+u+v=b$ and

$$
\begin{aligned}
& s^{2}+t^{2}+u^{2}+v^{2} \\
= & 2\left(\frac{s+t}{2}\right)^{2}+2\left(\frac{s-t}{2}\right)^{2}+2\left(\frac{u+v}{2}\right)^{2}+2\left(\frac{u-v}{2}\right)^{2} \\
= & 2\left(\frac{b+x}{4}\right)^{2}+2\left(\frac{y+z}{4}\right)^{2}+2\left(\frac{b-x}{4}\right)^{2}+2\left(\frac{y-z}{4}\right)^{2} \\
= & \frac{b^{2}+x^{2}+y^{2}+z^{2}}{4}=a .
\end{aligned}
$$

In view of Lemma 2.3 and the second inequality in (2.2), we have

$$
(|x|+|y|+|z|)^{2} \leqslant 3\left(x^{2}+y^{2}+z^{2}\right)=3\left(4 a-b^{2}\right)<(b+4)^{2} .
$$

Therefore

$$
s, t, u, v \geqslant \frac{b-|x|-|y|-|z|}{4}>-1
$$

and hence $s, t, u, v \in \mathbb{N}$.

Now we need one more lemma which is well known (cf. [1, p. 59]).

Lemma 2.5. For any $n \in \mathbb{Z}^{+}$, we have

$$
r_{4}(n)=8 \sum_{\substack{d \mid n \\ 4 \nmid d}} d
$$

where

$$
r_{4}(n):=\left|\left\{(w, x, y, z) \in \mathbb{Z}^{4}: w^{2}+x^{2}+y^{2}+z^{2}=n\right\}\right| .
$$

Proof of Theorem 1.1. (i) Let $I_{1}=[\alpha, \beta]$ be the interval given by (2.1). As $N \geqslant 7 \times 2^{2} m^{3}$, by Lemma 2.1 the length of the interval $I_{1}$ is greater than $2 m$. Choose $b_{0} \in\{\lceil\alpha\rceil+r: r=0, \ldots, m-1\}$ with $b_{0} \equiv N(\bmod m)$. Then $b_{1}=b_{0}+m \leqslant\lceil\alpha\rceil+2 m-1<\alpha+2 m<\beta$. Thus both $b_{0}$ and $b_{1}$ lie in $I_{1}$. Note that

$$
\frac{2}{m}\left(N-b_{1}\right)+b_{1}-\left(\frac{2}{m}\left(N-b_{0}\right)+b_{0}\right)=m-2 \equiv 2(\bmod 4) .
$$

So, for some $b \in\left\{b_{0}, b_{1}\right\}$ and $a=\frac{2}{m}(N-b)+b$ we have $2 \nmid a b$, or $2 \| a$ and $2 \mid b$. Obviously,

$$
b \geqslant \min I_{1}>0, a=\frac{2}{m} N+\left(1-\frac{2}{m}\right) b>0, \text { and } N=\frac{m}{2}(a-b)+b .
$$


Applying Lemmas 2.2 and 2.4, we see that there are $s, t, u, v \in \mathbb{N}$ satisfying (2.4). Therefore,

$$
\begin{aligned}
N & =\frac{m}{2}(a-b)+b \\
& =\frac{m}{2}\left(s^{2}-s+t^{2}-t+u^{2}-u+v^{2}-v\right)+s+t+u+v \\
& =p_{m+2}(s)+p_{m+2}(t)+p_{m+2}(u)+p_{m+2}(v) .
\end{aligned}
$$

(ii) Write $m=4 l$ with $l \in \mathbb{Z}^{+}$. Let $\varphi$ denote Euler's totient function. We want to show that none of the positive integers

$$
4 l^{2} \times \frac{4^{k \varphi(2 l+1)}-1}{2 l+1}(k=1,2, \ldots)
$$

can be written as $\sum_{i=1}^{4} p_{m+4}\left(x_{i}\right)$ with $x_{1}, x_{2}, x_{3}, x_{4} \in \mathbb{N}$.

Suppose that for some $n \in \mathbb{Z}^{+}$divisible by $\varphi(2 l+1)$ there are $w, x, y, z \in \mathbb{N}$ such that

$$
\begin{aligned}
4 l^{2} \times \frac{4^{n}-1}{2 l+1} & =p_{m+4}(w)+p_{m+4}(x)+p_{m+4}(y)+p_{m+4}(z) \\
& =\frac{4 l+2}{2}\left(w^{2}+x^{2}+y^{2}+z^{2}-w-x-y-z\right)+w+x+y+z .
\end{aligned}
$$

Then

$4^{n+1} l^{2}=((2 l+1) w-l)^{2}+((2 l+1) x-l)^{2}+((2 l+1) y-l)^{2}+((2 l+1) z-l)^{2}$.

As $r_{4}\left(4^{n+1} l^{2}\right)=r_{4}\left(4 l^{2}\right)$ by Lemma 2.5 , there are $w_{0}, x_{0}, y_{0}, z_{0} \in \mathbb{Z}$ with $w_{0}^{2}+x_{0}^{2}+y_{0}^{2}+z_{0}^{2}=4 l^{2}$ such that

$$
\begin{aligned}
& (2 l+1) w-l=2^{n} w_{0},(2 l+1) x-l=2^{n} x_{0}, \\
& (2 l+1) y-l=2^{n} y_{0},(2 l+1) z-l=2^{n} z_{0} .
\end{aligned}
$$

Since $2^{n} \equiv 1(\bmod 2 l+1)$ by Euler's theorem, we have

$$
w_{0} \equiv x_{0} \equiv y_{0} \equiv z_{0} \equiv-l(\bmod 2 l+1) \text {. }
$$

As $w_{0}^{2}+x_{0}^{2}+y_{0}^{2}+z_{0}^{2}=4 l^{2}$, we must have $w_{0}=x_{0}=y_{0}=z_{0}=-l$. Thus $(2 l+1) w=2^{n} w_{0}+l=l\left(1-2^{n}\right)<0$, which contradicts $w \in \mathbb{N}$.

\section{Proof of Theorem 1.2}

Lemma 3.1. Let l, $m, N \in \mathbb{Z}^{+}$with $N \geqslant 11 m^{2}(l m+1)$. Then the length of the interval

$$
I_{2}=\left[\frac{3}{2}+\sqrt{\frac{10 N}{m}-3}, 1+\sqrt{\frac{12 N}{m}}\right]
$$

is greater than $\mathrm{lm}$. 
Proof. The length $L_{2}$ of the interval $I_{2}$ is $\sqrt{12 x}-\sqrt{10 x-3}-1 / 2$, where $x=N / m \geqslant 11 \ln (\operatorname{lm}+1)$. Let $l_{0}=l m+1 / 2$. Then

$$
\begin{aligned}
L_{2}>l m & \Longleftrightarrow \sqrt{12 x}>\sqrt{10 x-3}+l_{0} \\
& \Longleftrightarrow 2 x+3-l_{0}^{2}+3>2 l_{0} \sqrt{10 x-3} \\
& \Longleftrightarrow 4 x\left(x-11 l_{0}^{2}+3\right)+\left(l_{0}^{2}-3\right)^{2}+12 l_{0}^{2}>0 .
\end{aligned}
$$

As

$$
x \geqslant 11 \operatorname{lm}(\operatorname{lm}+1)>11 l^{2} m^{2}+11 \operatorname{lm}+\frac{11}{4}-3=11 l_{0}^{2}-3,
$$

we have $L_{2}>l m$ as desired.

Lemma 3.2. Let $a, b, m, N \in \mathbb{Z}^{+}$with $m \geqslant 3$ and

$$
N=\frac{m}{2}(a-b)+b \geqslant \frac{3}{5} m \text {. }
$$

Suppose that $b$ belongs to the interval $I_{2}$ given by (3.1). Then

$$
b^{2}<6 a \text { and } 5 a<b^{2}+2 b+6 .
$$

Proof. Note that

$$
a=\left(1-\frac{2}{m}\right) b+\frac{2 N}{m} .
$$

Thus

$$
\begin{aligned}
b^{2}-6 a & =b^{2}-6\left(1-\frac{2}{m}\right) b-\frac{12 N}{m} \\
& =\left(b-3\left(1-\frac{2}{m}\right)\right)^{2}-9\left(1-\frac{2}{m}\right)^{2}-\frac{12 N}{m} .
\end{aligned}
$$

As $b \in I_{2}$, we have

$$
b \leqslant 1+\sqrt{\frac{12 N}{m}}<3\left(1-\frac{2}{m}\right)+\sqrt{9\left(1-\frac{2}{m}\right)^{2}+\frac{12 N}{m}}
$$

and hence $b^{2}-6 a<0$. On the other hand,

$$
\begin{aligned}
b^{2}+2 b+6-5 a & =b^{2}-\left(3-\frac{10}{m}\right) b+\left(6-\frac{10 N}{m}\right) \\
& =\left(b-\left(\frac{3}{2}-\frac{5}{m}\right)\right)^{2}-\left(\frac{3}{2}-\frac{5}{m}\right)^{2}+\left(6-\frac{10 N}{m}\right) \\
& >0
\end{aligned}
$$

since $(3 / 2-5 / m)^{2} \leqslant 9 / 4<3$ and

$$
b \geqslant \frac{3}{2}+\sqrt{\frac{10 N}{m}-3}>\frac{3}{2}-\frac{5}{m}+\sqrt{\left(\frac{3}{2}-\frac{5}{m}\right)^{2}-6+\frac{10 N}{m}} .
$$

This proves (3.2). 
Lemma 3.3. Let $a$ and $b$ be positive integers with $a \equiv b(\bmod 2)$ satisfying (3.2). Suppose that $2 \nmid a$ or $4 \| a$, and that $3 \mid a$ or $3 \nmid b$. Then there exist $s, t, u, v \in \mathbb{N}$ such that

$$
a=s^{2}+t^{2}+2 u^{2}+2 v^{2} \text { and } b=s+t+2 u+2 v .
$$

Proof. If $n \in \mathbb{N}$ is not of the form $4^{k}(8 l+7)$ with $k, l \in \mathbb{N}$, then by $(2.5)$ there are $x, u, v \in \mathbb{Z}$ with $u \equiv v(\bmod 2)$ such that

$$
n=x^{2}+u^{2}+v^{2}=x^{2}+2\left(\frac{u-v}{2}\right)^{2}+2\left(\frac{u+v}{2}\right)^{2} .
$$

We claim that there are $x, y, z \in \mathbb{Z}$ with $6 a-b^{2}=x^{2}+2 y^{2}+2 z^{2}$ such that all the numbers

$$
\begin{gathered}
s=\frac{b+x+2 y+2 z}{6}, \quad t=\frac{b-x-2 y+2 z}{6}, \\
u=\frac{b-x+y-z}{6}, \quad v=\frac{b+x-y-z}{6}
\end{gathered}
$$

are integral.

Case 1. $3 \nmid b$.

If $a \equiv b \equiv 1(\bmod 2)$, then $6 a-b^{2} \equiv 1(\bmod 4)$. When $4 \| a$ and $2 \mid b$, we have $6 a-b^{2} \equiv 4,8(\bmod 16)$. Thus $6 a-b^{2}=x^{2}+2 y^{2}+2 z^{2}$ for some $x, y, z \in \mathbb{Z}$. Clearly, $x \equiv b(\bmod 2)$ and $y \equiv z(\bmod 2)$. Note that

$$
x^{2}+2 y^{2}+2 z^{2} \equiv 6 a-b^{2} \equiv 2(\bmod 3) .
$$

As $x^{2} \not \equiv 2(\bmod 3)$, we have $3 \nmid y$ or $3 \nmid z$. Without loss of generality, we assume that $3 \nmid z$ and moreover $z \equiv b(\bmod 3)$. (If $z \equiv-b(\bmod 3)$ then $-z \equiv b(\bmod 3)$.$) As x^{2}-y^{2} \equiv x^{2}+2 y^{2} \equiv 0(\bmod 3)$, without loss of generality we may assume that $x \equiv y(\bmod 3)$. Now it is easy to see that all the numbers in (3.4) are integers.

Case 2. $3 \mid a$ and $3 \mid b$.

In this case, $a=3 a_{0}$ and $b=3 b_{0}$ for some $a_{0}, b_{0} \in \mathbb{Z}$. If $a \equiv b \equiv 1(\bmod 2)$, then $2 a_{0}-b_{0}^{2} \equiv 1(\bmod 4)$. When $4 \| a$ and $2 \mid b$, we have $2 a_{0}-b_{0}^{2} \equiv$ $4,8(\bmod 16)$. Thus $2 a_{0}-b_{0}^{2}=x_{0}^{2}+2 y_{0}^{2}+2 z_{0}^{2}$ for some $x_{0}, y_{0}, z_{0} \in \mathbb{Z}$. It follows that $x_{0} \equiv b_{0}(\bmod 2)$ and $y_{0} \equiv z_{0}(\bmod 2)$ since $a_{0} \equiv b_{0}(\bmod 2)$. Set $x=3 x_{0}, y=3 y_{0}$ and $z=3 z_{0}$. Then $6 a-b^{2}=9\left(2 a_{0}-b_{0}^{2}\right)=x^{2}+2 y^{2}+2 z^{2}$ and all the numbers in (3.4) are integers.

By the above, in either case, there are $x, y, z \in \mathbb{Z}$ for which $x^{2}+2 y^{2}+2 z^{2}=$ $6 a-b^{2}$ and $s, t, u, v \in \mathbb{Z}$, where $s, t, u, v$ are as in (3.4). Observe that

$$
s+t+2(u+v)=\frac{b+2 z}{3}+2 \times \frac{b-z}{3}=b
$$


and also

$$
\begin{aligned}
& s^{2}+t^{2}+2 u^{2}+2 v^{2} \\
= & 2\left(\frac{s+t}{2}\right)^{2}+2\left(\frac{s-t}{2}\right)^{2}+(u+v)^{2}+(u-v)^{2} \\
= & 2\left(\frac{b+2 z}{6}\right)^{2}+2\left(\frac{x+2 y}{6}\right)^{2}+\left(\frac{b-z}{3}\right)^{2}+\left(\frac{x-y}{3}\right)^{2} \\
= & \frac{b^{2}+x^{2}+2 y^{2}+2 z^{2}}{6}=a .
\end{aligned}
$$

In view of Lemma 2.3 and (3.2),

$$
(|x|+2|y|+2|z|)^{2} \leqslant 5\left(x^{2}+2 y^{2}+2 z^{2}\right)=5\left(6 a-b^{2}\right)<(b+6)^{2}
$$

and hence

$$
b-|x|-2|y|-2|z|>-6 .
$$

So we have

$$
s, t, u, v \geqslant \frac{b-|x|-2|y|-2|z|}{6}>-1,
$$

and hence $s, t, u, v \in \mathbb{N}$.

In view of the above, we have completed the proof of Lemma 3.3.

Remark 3.1. For $s, t, u, v$ given in (3.4), the identity

$$
\begin{aligned}
6\left(s^{2}+t^{2}+2 u^{2}+2 v^{2}\right)= & b^{2}+x^{2}+2 y^{2}+2 z^{2} \\
= & (s+t+2 u+2 v)^{2}+(s-t-2 u+2 v)^{2} \\
& +2(s-t+u-v)^{2}+2(s+t-u-v)^{2}
\end{aligned}
$$

is a special case of our following general identity

$$
\begin{aligned}
& (a+b)(c+d)\left(w^{2}+a b x^{2}+c d y^{2}+a b c d z^{2}\right) \\
= & a c(w+b x+d y+b d z)^{2}+a d(w+b x-c y-b c z)^{2} \\
& +b c(w-a x+d y-a d z)^{2}+b d(w-a x-c y+a c z)^{2} .
\end{aligned}
$$

We have also found another similar identity:

$$
\begin{aligned}
& (3 b+4)\left(w^{2}+2 x^{2}+(b+1) y^{2}+2 b z^{2}\right) \\
= & (w+2 x+(b+1) y+2 b z)^{2}+2(w-(b+1) y+b z)^{2} \\
& +(b+1)(w-2 x+y)^{2}+2 b(w+x-2 z)^{2} .
\end{aligned}
$$

Proof of Theorem 1.2. (i) Let $I_{2}=[\alpha, \beta]$ be the interval given by (3.1). As $N \geqslant 1628 m^{3}=(12+1 / 3) m \times 132 m^{2} \geqslant 11 m^{2} \times 12(12 m+1)$, by Lemma 3.1 the length of the interval $I_{2}$ is greater than $12 \mathrm{~m}$. We distinguish two cases to construct integers $b \in I_{2}$ and $a \equiv b(\bmod 2)$ for which $N=\frac{m}{2}(a-b)+b$, and $2 \nmid a$ or $4 \| a$, and $3 \mid a$ or $3 \nmid b$. 
Case $1.3 \nmid m$ or $3 \nmid N$.

Choose $b_{0} \equiv N(\bmod m)$ with $b_{0} \in\{\lceil\alpha\rceil+r: r=0, \ldots, m-1\}$, and let $b_{j}=b_{0}+j m$ for $j=1, \ldots, 7$. Since $\lceil\alpha\rceil+8 m-1<\alpha+8 m<\beta$, we have $b_{i} \in I_{2}$ for all $i=0, \ldots, 7$.

If $2 \nmid m$, then we choose $i \in\{0,1\}$ with $b_{i}$ odd. When $4 \mid m$, we may choose $i \in\{0,1,2,3\}$ with

$$
a_{i}:=\frac{2}{m}\left(N-b_{i}\right)+b_{i} \equiv 4(\bmod 8)
$$

since $a_{i}-a_{0}=-2 i+i m=2 i(m / 2-1)$ with $m / 2-1$ odd. If $3 \mid m$ and $3 \nmid N$, then $b=b_{i} \equiv N \not \equiv 0(\bmod 3)$. When $3 \nmid m$, we choose $j \in\{i, i+4\}$ with $b=b_{j} \not \equiv 0(\bmod 3)$, and note that

$$
a_{i+4}-a_{i}=4 m-8 \equiv \begin{cases}0(\bmod 2) & \text { if } 2 \nmid m, \\ 0(\bmod 8) & \text { if } 4 \mid m .\end{cases}
$$

As $N \equiv b(\bmod m)$, we see that $a=2(N-b) / m+b$ is an integer with $a \equiv b(\bmod 2)$. By our choice, $2 \nmid b$ if $2 \nmid m$, and $a \equiv 4(\bmod 8)$ if $4 \mid m$. Note also that $3 \nmid b$.

Case $2 . m \equiv N \equiv 0(\bmod 3)$.

Choose $b_{0} \in\{\lceil\alpha\rceil+r: r=0,1, \ldots, 3 m-1\}$ with $b_{0} \equiv N(\bmod 3 m)$. If $2 \nmid m$, then we choose $b \in\left\{b_{0}, b_{0}+3 m\right\}$ with $b$ odd and hence $a=$ $\frac{2}{m}(N-b)+b \equiv b \equiv 1(\bmod 2)$. When $4 \mid m$, we may choose $b \in\left\{b_{0}+3 j m\right.$ : $j=0,1,2,3\}$ with $a=\frac{2}{m}(N-b)+b \equiv 4(\bmod 8)$, for

$$
\frac{2}{m}\left(N-b_{0}-3 j m\right)+b_{0}+3 j m-\left(\frac{2}{m}\left(N-b_{0}\right)+b_{0}\right)=6 j\left(\frac{m}{2}-1\right)
$$

with $m / 2-1$ odd. Note that

$$
\alpha \leqslant b_{0}<b_{0}+9 m \leqslant\lceil\alpha\rceil+3 m-1+9 m<\alpha+12 m<\beta
$$

and hence $b \in I_{2}$. Obviously, $a \equiv b \equiv N \equiv 0(\bmod 3)$.

Now we have constructed positive integers $b \in I_{2}$ and $a \equiv b(\bmod 2)$ with $N=\frac{m}{2}(a-b)+b$ such that $2 \nmid a$ or $4 \| a$, and $3 \mid a$ or $3 \nmid b$. So (3.2) holds by Lemma 3.2. In view of Lemma 3.3, (3.3) holds for some $s, t, u, v \in \mathbb{N}$. Therefore,

$$
\begin{aligned}
N & =\frac{m}{2}\left(s^{2}+t^{2}+2 u^{2}+2 v^{2}-s-t-2 u-2 v\right)+s+t+2 u+2 v \\
& =m\left(\begin{array}{l}
s \\
2
\end{array}\right)+m\left(\begin{array}{l}
t \\
2
\end{array}\right)+2 m\left(\begin{array}{l}
u \\
2
\end{array}\right)+2 m\left(\begin{array}{l}
v \\
2
\end{array}\right)+s+t+2 u+2 v \\
& =p_{m+2}(s)+p_{m+2}(t)+2 p_{m+2}(u)+2 p_{m+2}(v) .
\end{aligned}
$$

This proves part (i) of Theorem 1.2. 
(ii) Now assume that $m=2 l$ with $l \in \mathbb{Z}^{+}$odd. We want to show that none of the positive integers

$$
(l-1)^{2} \times \frac{4^{k \varphi(l)}-1}{l}(k=1,2,3, \ldots)
$$

can be written as $p_{m+2}(w)+p_{m+2}(x)+2 p_{m+2}(y)+2 p_{m+2}(z)$ with $w, x, y, z \in$ $\mathbb{N}$.

Let $n \in \mathbb{Z}^{+}$be a multiple of $\varphi(l)$. Then $2^{n} \equiv 1(\bmod l)$ by Euler's theorem. Suppose that there are $w, x, y, z \in \mathbb{N}$ for which

$$
\begin{aligned}
& (l-1)^{2} \times \frac{4^{n}-1}{l} \\
= & p_{m+2}(w)+p_{m+2}(x)+2 p_{m+2}(y)+2 p_{m+2}(z) \\
= & \frac{2 l}{2}\left(w^{2}+x^{2}+2 y^{2}+2 z^{2}-w-x-2 y-2 z\right)+w+x+2 y+2 z .
\end{aligned}
$$

Then we have

$$
\begin{aligned}
& 4^{n+1}(l-1)^{2} \\
= & (2 l w-(l-1))^{2}+(2 l x-(l-1))^{2}+2(2 l y-(l-1))^{2}+2(2 l z-(l-1))^{2} \\
= & (2 l w-(l-1))^{2}+(2 l x-(l-1))^{2}+(2 l(y+z)-2(l-1))^{2}+(2 l(y-z))^{2}
\end{aligned}
$$

and hence

$4^{n}(l-1)^{2}=\left(l w-\frac{l-1}{2}\right)^{2}+\left(l x-\frac{l-1}{2}\right)^{2}+(l(y+z-1)+1)^{2}+(l(y-z))^{2}$.

As $4 \mid(l-1)^{2}$, by Lemma 2.5 we have $r_{4}\left(4^{n}(l-1)^{2}\right)=r_{4}\left((l-1)^{2}\right)$. So there are $w_{0}, x_{0}, y_{0}, z_{0} \in \mathbb{Z}$ with

$$
w_{0}^{2}+x_{0}^{2}+y_{0}^{2}+z_{0}^{2}=(l-1)^{2}
$$

such that

$l w-\frac{l-1}{2}=2^{n} w_{0}, l x-\frac{l-1}{2}=2^{n} x_{0}, l(y+z-1)+1=2^{n} y_{0}, l(y-z)=2^{n} z_{0}$.

As $2^{n} \equiv 1(\bmod l)$, we see that

$$
w_{0} \equiv \frac{1-l}{2}(\bmod l), x_{0} \equiv \frac{1-l}{2}(\bmod l), y_{0} \equiv 1(\bmod l), z_{0} \equiv 0(\bmod l) \text {. }
$$

Observe that $l=m / 2 \geqslant 2$ and hence $w_{0} x_{0} \neq 0$. Thus $y_{0}^{2}+z_{0}^{2} \leqslant(l-1)^{2}-2$. Since $y_{0} \equiv 1(\bmod l)$ and $z_{0} \equiv 0(\bmod l)$, we must have $y_{0}=1$ and $z_{0}=0$. Now (3.7) yields $w_{0}^{2}+x_{0}^{2}=(l-1)^{2}-1=l^{2}-2 l$. As $w_{0} \equiv x_{0} \equiv$ $(1-l) / 2(\bmod l)$, we must have $\left\{w_{0}, x_{0}\right\} \subseteq\{(1-l) / 2,(1+l) / 2\}$. It is easy to verify directly that none of the numbers

$$
\left(\frac{1-l}{2}\right)^{2}+\left(\frac{1+l}{2}\right)^{2}, 2\left(\frac{l-1}{2}\right)^{2}, 2\left(\frac{1+l}{2}\right)^{2}
$$


is equal to $(l-1)^{2}-1=l^{2}-2 l$. This contradiction concludes the proof of Theorem 1.2(ii).

\section{Proof of Theorem 1.3}

Lemma 4.1. Let $a$ and $b$ be positive integers satisfying (3.2) for which $a \equiv b(\bmod 2)$, and $a \equiv 3(\bmod 9)$ or $3 \nmid b$. Then there exist $s, t, u, v \in \mathbb{N}$ such that

$$
a=s^{2}+t^{2}+u^{2}+3 v^{2} \text { and } b=s+t+u+3 v .
$$

Proof. It is known that (cf. Dickson [3, pp.112-113])

$$
\left\{x^{2}+y^{2}+3 z^{2}: x, y, z \in \mathbb{Z}\right\}=\mathbb{N} \backslash\left\{9^{k}(9 l+6): k, l \in \mathbb{N}\right\} .
$$

If $3 \nmid b$, then $6 a-b^{2} \equiv 2(\bmod 3)$. If $a \equiv 3(\bmod 9)$ and $3 \mid b$, then $6 a-b^{2} \equiv \pm 9(\bmod 27)$. By $(4.2), 6 a-b^{2}=x^{2}+y^{2}+3 z^{2}$ for some $x, y, z \in \mathbb{Z}$. Clearly, $x^{2}+y^{2} \equiv 2 b^{2}(\bmod 3)$. Without loss of generality, we may assume that $x \equiv y \equiv b(\bmod 3)$. (If $x \equiv-b(\bmod 3)$ then $-x \equiv b(\bmod 3)$.) If $a$ and $b$ are both odd, then $x^{2}+y^{2}+3 z^{2}=6 a-b^{2} \equiv 1(\bmod 4)$, and hence one of $x$ and $y$ is odd. If $a$ and $b$ are both even, then $x^{2}+y^{2}+3 z^{2}=6 a-b^{2} \equiv$ $0(\bmod 4)$, and hence one of $x$ and $y$ is even. Without loss of generality, we may assume that $x \equiv a \equiv b(\bmod 2)$ and $y \equiv z(\bmod 2)$. Thus all the numbers

$$
s=\frac{b+x+y+3 z}{6}, t=\frac{b+x+y-3 z}{6}, u=\frac{b+x-2 y}{6}, v=\frac{b-x}{6}
$$

are integers. Observe that

$$
s+t+u+3 v=\frac{b+x}{2}+3 \times \frac{b-x}{6}=b
$$

and

$$
\begin{aligned}
& s^{2}+t^{2}+u^{2}+3 v^{2} \\
= & 2\left(\frac{s+t}{2}\right)^{2}+2\left(\frac{s-t}{2}\right)^{2}+u^{2}+3 v^{2} \\
= & 2\left(\frac{b+x+y}{6}\right)^{2}+2\left(\frac{z}{2}\right)^{2}+\left(\frac{b+x-2 y}{6}\right)^{2}+3\left(\frac{b-x}{6}\right)^{2} \\
= & \frac{b^{2}+x^{2}+y^{2}+3 z^{2}}{6}=a .
\end{aligned}
$$

In view of Lemma 2.3 and (3.2),

$$
(|x|+|y|+3|z|)^{2} \leqslant 5\left(x^{2}+y^{2}+3 z^{2}\right)=5\left(6 a-b^{2}\right)<(b+6)^{2}
$$


and hence

$$
b-|x|-|y|-3|z|>-6 .
$$

So we have

$$
s, t, u, v \geqslant \frac{b-|x|-|y|-3|z|}{6}>-1
$$

and hence $s, t, u, v \in \mathbb{N}$. This concludes the proof.

Proof of Theorem 1.3. As

$$
N \geqslant 924 m^{3}=99 m^{3}\left(9+\frac{1}{3}\right) \geqslant 99 m^{3}\left(9+\frac{1}{m}\right)=99 m^{2}(9 m+1),
$$

the length of the interval $I_{2}=[\alpha, \beta]$ defined in Lemma 3.1 is greater than $9 m$.

Let $b_{0} \in\{\lceil\alpha\rceil+r: r=0,1, \ldots, m-1\}$ with $b_{0} \equiv N(\bmod m)$. If $3 \nmid m$ or $3 \nmid N$, then we may choose $b \in\left\{b_{0}, b_{0}+m\right\}$ with $b \neq \equiv(\bmod 3)$. When $3 \mid m$ and $3 \mid N$, we let $c_{0} \in\{\lceil\alpha\rceil+r: r=0, \ldots, 3 m-1\}$ with $c_{0} \equiv N(\bmod 3 m)$, and set $b=c_{0}+j 3 m$ with $j \in\{0,1,2\}$ such that

$$
\frac{2}{m}\left(N-c_{0}\right)+c_{0}-6 j \equiv 3(\bmod 9) \text {. }
$$

Note that $b \in I_{2}$ since

$$
\alpha \leqslant b \leqslant\lceil\alpha\rceil+3 m-1+6 m<\alpha+9 m<\beta .
$$

Let

$$
a=\frac{2}{m}(N-b)+b, \text { i.e., } N=\frac{m}{2}(a-b)+b .
$$

Then

$$
a=\frac{2}{m} N+\left(1-\frac{2}{m}\right) b>0 \quad \text { and } \quad a \equiv b(\bmod 2) .
$$

If $3 \mid b$, then $3 \mid m$ and

$a=\frac{2}{m}(N-b)+b=\frac{2}{m}\left(N-c_{0}-3 j m\right)+c_{0}+3 j m \equiv \frac{2}{m}\left(N-c_{0}\right)-6 j \equiv 3(\bmod 9)$.

By Lemmas 3.2 and 4.1, there are $s, t, u, v \in \mathbb{N}$ satisfying (4.1). Therefore,

$$
\begin{aligned}
N & =\frac{m}{2}\left(s^{2}+t^{2}+u^{2}+3 v^{2}-s-t-u-3 v\right)+s+t+u+3 v \\
& =p_{m+2}(s)+p_{m+2}(t)+p_{m+2}(u)+3 p_{m+2}(v) .
\end{aligned}
$$

This completes the proof of Theorem 1.3. 
Lemma 5.1. Let $l, m, N \in \mathbb{Z}^{+}$with $l m \geqslant 20$ and $N \geqslant 3 \operatorname{lm}^{2}(5 \operatorname{lm}+12)$. Then the length of the interval

$$
I_{3}=\left[\frac{5}{2}+\sqrt{\frac{14 N}{m}-1}, \frac{4}{3}+4 \sqrt{\frac{N}{m}}\right]
$$

is greater than $\mathrm{lm}$.

Proof. The length of the interval $I_{3}$ is $4 \sqrt{x}-\sqrt{14 x-1}-7 / 6$, where $x=$ $N / m$. Set $l_{0}=l m+7 / 6$. Then

$$
\begin{aligned}
& 4 \sqrt{x}-\sqrt{14 x-1}-\frac{7}{6}>\operatorname{lm} \\
\Longleftrightarrow & 4 \sqrt{x}>\sqrt{14 x-1}+l_{0} \\
\Longleftrightarrow & 2 x+1-l_{0}^{2}>2 l_{0} \sqrt{14 x-1} \\
\Longleftrightarrow & 4 x\left(x+1-15 l_{0}^{2}\right)+\left(l_{0}^{2}-1\right)^{2}+4 l_{0}^{2}>0 .
\end{aligned}
$$

Note that

$$
x \geqslant 15 l^{2} m^{2}+36 l m>15 l^{2} m^{2}+35 l m+\frac{245}{12}-1=15 l_{0}^{2}-1 .
$$

So the desired result follows.

Lemma 5.2. Let $a, b, m, N$ be positive integers with $m \geqslant 3$,

$$
N=\frac{m}{2}(a-b)+b \geqslant \frac{4}{7} m
$$

and $b \in I_{3}$, where $I_{3}$ is the interval given by (5.1). Then

$$
b^{2}<8 a \text { and } 7 a<b^{2}+2 b+8 .
$$

Proof. Clearly,

$$
a=\left(1-\frac{2}{m}\right) b+\frac{2 N}{m}
$$

Thus

$$
\begin{aligned}
b^{2}-8 a & =b^{2}-8\left(1-\frac{2}{m}\right) b-\frac{16 N}{m} \\
& =\left(b-4\left(1-\frac{2}{m}\right)\right)^{2}-16\left(\left(1-\frac{2}{m}\right)^{2}+\frac{N}{m}\right) .
\end{aligned}
$$

As $b \in I_{3}$, we have

$$
b \leqslant \frac{4}{3}+4 \sqrt{\frac{N}{m}}<4\left(1-\frac{2}{m}\right)+4 \sqrt{\left(1-\frac{2}{m}\right)^{2}+\frac{N}{m}}
$$


and hence $b^{2}-8 a<0$. On the other hand,

$$
\begin{aligned}
& b^{2}+2 b+8-7 a \\
= & b^{2}-\left(5-\frac{14}{m}\right) b+\left(8-\frac{14 N}{m}\right) \\
= & \left(b-\left(\frac{5}{2}-\frac{7}{m}\right)\right)^{2}-\left(\left(\frac{5}{2}-\frac{7}{m}\right)^{2}+\frac{14 N}{m}-8\right)>0
\end{aligned}
$$

since $(5 / 2-7 / m)^{2} \leqslant 25 / 4<7$ and

$$
b \geqslant \frac{5}{2}+\sqrt{\frac{14 N}{m}-1}>\frac{5}{2}-\frac{7}{m}+\sqrt{\left(\frac{5}{2}-\frac{7}{m}\right)^{2}+\frac{14 N}{m}-8 .}
$$

Therefore (5.2) holds.

Lemma 5.3. Let $a$ and $b$ be positive integers satisfying (5.2). Then there are $s, t, u, v \in \mathbb{N}$ such that

$$
a=s^{2}+t^{2}+2 u^{2}+4 v^{2} \text { and } b=s+t+2 u+4 v,
$$

under one of the following conditions (i)-(iii):

(i) $2 \nmid a b$.

(ii) $2 \mid a$ and $2 \| b$.

(iii) $4 \mid a$ and $4 \| b$, or $a \equiv b+4(\bmod 16)$ and $8 \mid b$.

Proof. It is known (cf. [3, pp. 112-113]) that

$$
\left\{x^{2}+2 y^{2}+4 z^{2}: x, y, z \in \mathbb{Z}\right\}=\mathbb{N} \backslash\left\{4^{k}(16 l+14): k, l \in \mathbb{N}\right\} .
$$

We claim that if one of (i)-(iii) holds then $8 a-b^{2}=x^{2}+2 y^{2}+4 z^{2}$ for some $x, y, z \in \mathbb{Z}$ such that all the numbers

$$
u=\frac{b+x-2 y}{8}, v=\frac{b-x}{8}, s=u+\frac{y+z}{2}, t=u+\frac{y-z}{2}
$$

are integers.

Case 1. $2 \nmid a b$.

Since $8 a-b^{2} \equiv-1(\bmod 8)$, we have $8 a-b^{2}=x^{2}+2 y^{2}+4 z^{2}$ for some $x, y, z \in \mathbb{Z}$. As $x^{2}+2 y^{2} \equiv-1(\bmod 4)$, we have $2 \nmid x y$. Since $4 z^{2} \equiv-b^{2}-x^{2}-2 y^{2} \equiv-1-1-2(\bmod 8)$, we also have $2 \nmid z$. Note that

$$
x^{2}=8 a-b^{2}-2 y^{2}-4 z^{2} \equiv 8-b^{2}-2-4=2-b^{2} \equiv b^{2}(\bmod 16)
$$

and hence $x \equiv \pm b(\bmod 8)$. Without loss of generality, we may assume that $x \equiv b(\bmod 8)$ and $y \equiv b(\bmod 4) .($ If $y \equiv-b(\bmod 4)$ then $-y \equiv b(\bmod 4)$.) Thus all the four numbers in (5.5) are integers. 
Case 2. $2 \mid a$ and $2 \| b$.

Write $a=2 a_{0}$ and $b=2 b_{0}$ with $a_{0}, b_{0} \in \mathbb{Z}$ and $2 \nmid b_{0}$. Since $4 a_{0}-b_{0}^{2} \equiv$ $3(\bmod 4)$, by $(5.4)$ we have $4 a_{0}-b_{0}^{2}=x_{0}^{2}+2 y_{0}^{2}+4 z_{0}^{2}$ for some $x_{0}, y_{0}, z_{0} \in \mathbb{Z}$. As $x_{0}^{2}+2 y_{0}^{2} \equiv 3(\bmod 4)$, both $x_{0}$ and $y_{0}$ are odd. Without loss of generality, we may assume that $x_{0} \equiv y_{0} \equiv b_{0}(\bmod 4)$. Set $x=2 x_{0}, y=2 y_{0}$ and $z=2 z_{0}$. Then

$$
8 a-b^{2}=4\left(4 a_{0}-b_{0}^{2}\right)=x^{2}+2 y^{2}+4 z^{2}
$$

and all the numbers in (5.5) are integers.

Case 3. $4 \mid a$ and $4 \| b$, or $a \equiv b+4(\bmod 16)$ and $8 \mid b$.

Write $a=4 a_{0}$ and $b=4 b_{0}$ with $a_{0}, b_{0} \in \mathbb{Z}$. Then

$$
2 a_{0}-b_{0}^{2} \equiv \begin{cases}1(\bmod 2) & \text { if } 4 \| b\left(\text { i.e., } 2 \nmid b_{0}\right), \\ 2\left(b_{0}+1\right)-b_{0}^{2} \equiv 2(\bmod 8) & \text { if } a \equiv b+4(\bmod 16) \text { and } 8 \mid b .\end{cases}
$$

Thus, by (5.4) we have $2 a_{0}-b_{0}^{2}=x_{0}^{2}+2 y_{0}^{2}+4 z_{0}^{2}$ for some $x_{0}, y_{0}, z_{0} \in \mathbb{Z}$. Obviously $x_{0} \equiv b_{0}(\bmod 2)$. Set $x=4 x_{0}, y=4 y_{0}$ and $z=4 z_{0}$. Then all the numbers in (5.5) are integers.

Now assume that one of the conditions (i)-(iii) holds. By the claim we have proved, there are $x, y, z \in \mathbb{Z}$ such that $8 a-b^{2}=x^{2}+2 y^{2}+4 z^{2}$ and also $s, t, u, v \in \mathbb{Z}$, where $s, t, u, v$ are given by (5.5). Clearly,

$$
s+t+2 u+4 v=y+2 u+2 u+4 v=\frac{b+x}{2}+\frac{b-x}{2}=b
$$

and

$$
\begin{aligned}
& s^{2}+t^{2}+2 u^{2}+4 v^{2} \\
= & 2\left(u+\frac{y}{2}\right)^{2}+2\left(\frac{z}{2}\right)^{2}+2\left(\frac{b+x-2 y}{8}\right)^{2}+4\left(\frac{b-x}{8}\right)^{2} \\
= & 2\left(\frac{b+x+2 y}{8}\right)^{2}+2\left(\frac{b+x-2 y}{8}\right)^{2}+\frac{z^{2}}{2}+\left(\frac{b-x}{4}\right)^{2} \\
= & \left(\frac{b+x}{4}\right)^{2}+\left(\frac{y}{2}\right)^{2}+\frac{z^{2}}{2}+\left(\frac{b-x}{4}\right)^{2} \\
= & \frac{b^{2}+x^{2}+2 y^{2}+4 z^{2}}{8}=a .
\end{aligned}
$$

In view of Lemma 2.3 and (5.2),

$$
(|x|+2|y|+4|z|)^{2} \leqslant 7\left(x^{2}+2 y^{2}+4 z^{2}\right)=7\left(8 a-b^{2}\right)<(b+8)^{2}
$$

and hence

$$
b-|x|-2|y|-4|z|>-8 .
$$


So we have

$$
u, v, s, t \geqslant \frac{b-|x|-2|y|-4|z|}{8}>-1
$$

and hence $s, t, u, v \in \mathbb{N}$.

In view of the above, we have completed the proof of Lemma 5.3.

Proof of Theorem 1.4. As

$$
N \geqslant 96 m^{2} \times 11 m \geqslant 96 m^{2}(10 m+3)=24 m^{2}(40 m+12),
$$

applying Lemma 5.1 with $l=8$ we find that the interval $I_{3}=[\alpha, \beta]$ given by (5.1) has length greater than $8 m$.

Case 1. $4 \nmid m$ or $8 \nmid N$.

Choose $b_{0} \in\{\lceil\alpha\rceil+r: r=0, \ldots, m-1\}$ with $N \equiv b_{0}(\bmod m)$. Set $b_{1}=b_{0}+m$. Then

$$
\alpha \leqslant b_{0}<b_{1} \leqslant\lceil\alpha\rceil+2 m-1<\alpha+8 m<\beta
$$

and thus $b_{j} \in I_{3}$ for each $j=0,1$. Note that

$$
a_{j}:=\frac{2}{m}\left(N-b_{j}\right)+b_{j}=\frac{2}{m}\left(N-b_{0}\right)+b_{0}+(m-2) j .
$$

If $2 \nmid m$, then $a_{j} \equiv b_{j} \equiv 1(\bmod 2)$ for some $j \in\{0,1\}$. If $2 \mid m$ and $2 \nmid N$, then $a_{0} \equiv b_{0} \equiv 1(\bmod 2)$.

If $2 \| m$ and $2 \mid N$, then for some $j \in\{0,1\}$ we have $b_{j} \equiv 2(\bmod 4)$ and $2 \mid$ $a_{j}$. When $4 \mid m$ and $2 \| N$, we have $b_{0} \equiv 2(\bmod 4)$ and $a_{0} \equiv b_{0} \equiv 0(\bmod 2)$. If $4 \| m$ and $4 \| N$, then $4 \mid b_{0}$, and for some $j \in\{0,1\}$ we have $b_{j} \equiv 4(\bmod 8)$ and $a_{j} \equiv b_{j} \equiv 0(\bmod 4)$. When $8 \mid m$ and $4 \| N$, we have $b_{0} \equiv 4(\bmod 8)$ and $a_{0} \equiv b_{0}(\bmod 2)$, hence for some $j \in\{0,1\}$ we have $a_{j} \equiv 0(\bmod 4)$ and $b_{j} \equiv b_{0} \equiv 4(\bmod 8)$.

Case 2. $4 \mid m$ and $8 \mid N$.

Choose $b \in\{\lceil\alpha\rceil+r: r=0, \ldots, 8 m-1\}$ such that $b \equiv N-2 m(\bmod 8 m)$. Since $\alpha \leqslant b \leqslant\lceil\alpha\rceil+8 m-1<\alpha+8 m<\beta$, we have $b \in I_{3}$. Clearly, $8 \mid b$ since $8 \mid N$ and $4 \mid m$. Note that

$$
\frac{2}{m}(N-b)+b \equiv 4+b(\bmod 8) \text {. }
$$

By the above, in either case we can always find $b \in I_{3}$ and $a \in \mathbb{Z}$ for which one of (i)-(iii) in Lemma 5.3 holds and also

$$
a=\frac{2}{m}(N-b)+b, \text { i.e., } N=\frac{m}{2}(a-b)+b .
$$


By Lemmas 5.2 and 5.3, there are $s, t, u, v \in \mathbb{N}$ satisfying (5.3). Therefore,

$$
\begin{aligned}
N & =\frac{m}{2}\left(s^{2}+t^{2}+2 u^{2}+4 v^{2}-s-t-2 u-4 v\right)+s+t+2 u+4 v \\
& =p_{m+2}(s)+p_{m+2}(t)+2 p_{m+2}(u)+4 p_{m+2}(v) .
\end{aligned}
$$

In view of the above, we have finished the proof of Theorem 1.4.

Acknowledgements. This research was supported by the National Natural Science Foundation of China (grant no. 11571162).

\section{REFERENCES}

[1] B. C. Berndt, Number Theory in the Spirit of Ramanujan, Amer. Math. Soc., Providence, R.I., 2006.

[2] L. E. Dickson, Quaternary quadratic forms representing all integers, Amer. J. Math. 49 (1927), 39-56.

[3] L. E. Dickson, Modern Elementary Theory of Numbers, University of Chicago Press, Chicago, 1939.

[4] F. Ge and Z.-W. Sun, On some universal sums of generalized polygonal numbers, Colloq. Math. 145 (2016), 149-155.

[5] R. K. Guy, Every number is expressible as the sum of how many polygonal numbers? Amer. Math. Monthly 101 (1994), 169-172.

[6] D. Krachun, On sums of triangular numbers, preprint, arXiv:1602.01133, (2016).

[7] C. J. Moreno and S. S. Wagstaff, Sums of Squares of Integers, Chapman \& Hall/CRC, Boca Raton, FL, 2006.

[8] M. B. Nathanson, A short proof of Cauchy's polygonal number theorem, Proc. Amer. Math. Soc. 99 (1987), 22-24.

[9] M. B. Nathanson, Additive Number Theory: The Classical Bases, Grad. Texts in Math., Vol. 164, Springer, New York, 1996.

[10] S. Ramanujan, On the expression of a number in the form $a x^{2}+b y^{2}+c z^{2}+d w^{2}$, Proc. Cambridge Philos. Soc. 19 (1917), 11-21.

[11] Z.-W. Sun, On universal sums of polygonal numbers, Sci. China Math. 58 (2015), 1367-1396.

[12] Z.-W. Sun, A result similar to Lagrange's theorem, J. Number Theory 162 (2016), 190-211.

(Xiang-Zi Meng) Department of Mathematics, Nanjing University, NanJing 210093, PeOple's Republic of China

E-mail address: xzmeng@smail.nju.edu.cn

(Zhi-Wei Sun, corresponding author) Department of Mathematics, NanJing University, NAnjing 210093, People's Republic of China

E-mail address: zwsun@nju.edu.cn 